\title{
Aproximación política a la implementación de la gestión ambiental en América Latina desde los Objetivos de Desarrollo del Milenio
}

\author{
A political approach to environmental management deployment in Latin America \\ according to the Millennium Development Goals
}

Recibido: 08/09/13 - Aprobado versión final: 25/09/14

Wilinthon James Osorio Vélez*

\begin{abstract}
Resumen: este artículo examina algunas posiciones económicas que contrastan con la perspectiva ambientalista, y plantea cómo estas posiciones interactúan entre sí para hacer una lectura actualizada de las iniciativas que en esta materia se desarrollan en América Latina, como la gestión que realizan los gobiernos de la región a fin de cumplir con los compromisos pactados en la Cumbre del Milenio. Del mismo modo, el artículo esboza una perspectiva global sobre iniciativas populares en esta materia y ofrece un criterio de participación del saber contable sobre el control, la información y el ambiente, que proporcione herramientas mínimas de aportación de la disciplina al cumplimiento de estos objetivos.
\end{abstract}

Palabras clave: globalización, medio ambiente y desarrollo, Objetivos de Desarrollo del Milenio, cambio climático.

\begin{abstract}
: this paper discusses several economic standpoints that are in contrast with the environmentalist approach, and argues how such standpoints interact with each other to allow for an updated reading of related ongoing initiatives across Latin America, such as management advanced by the region's governments in order to achieve the goals agreed at the Millennium Summit. Similarly, this paper draws a global perspective on grassroots related initiatives, and provides a criteria for the participation of accounting knowledge on control, information and environment, providing basic tools to achieve these goals.
\end{abstract}

Keywords: globalization, environment and development, Millennium Development Goals, climate change.

JEL: Q56

* Contador Público y Magister ( C en Tecnología e Ingeniería Ambiental. Docente del Politécnico Colombiano Jaime Isaza Cadavid, Medellín - Colombia. nuevosescribanos@gmail.com 


\section{Contekto osorio, w.}

\section{Considérations politiques sur la mise en place de la gestion de l'environnement en Amérique Latine dans le cadre des Objectifs du Millénaire pour le Développement}

Résumé: dans cet article nous examinons quelques approches économiques qui vont à l'encontre de la perspective environnementaliste ; aussi il est question de démontrer comment ces approches-là interagissent. Enfin nous faisons une lecture actuelle des initiatives que dans le domaine environnemental sont entreprises en Amérique Latine, notamment en ce qui concerne l'action des gouvernements visant à respecter les engagements contractés lors du Sommet du Millénaire ; il sera question aussi de porter un regard global sur les initiatives populaires dans ce domaine et d'offrir un critère de participation du savoir comptable à propos du contrôle, de l'information et de l'environnement fournissant des outils de base afin de permettre d'atteindre ces objectifs-là.

Mots clé: globalisation, environnement et développement, Objectifs du Millénaire pour le Développement, changement climatique.

\section{Introducción}

Una manera de observar la realidad económica que circunda los contextos sociales está determinada por la forma como se asumen con objetividad, tanto de manera individual como colectiva, los efectos que la humanidad provoca en cada época sobre la evolución social y cultural. Ha faltado rigurosidad para fundar la elaboración de estudios concretos sobre los modos de producción, asociados a los procesos de transformación y de apropiación de la naturaleza (Leff, 1986, p. 125); no es gratuito que cuando se realiza una mirada historiográfica de la especie, dicha mirada siempre esté asociada a un modo de producción y no pueda ser ajena a estos tiempos. La economía ortodoxa ha sido incapaz de integrar las externalidades de los procesos productivos en sus paradigmas teóricos y en sus prácticas de planificación. La pregunta entonces que debe generarse en los círculos académicos actuales debería ser cómo se está viviendo este tiempo y cuál será el legado económico que va a dar cuenta del ser humano de hoy en el futuro.

Esta mirada puede estar asociada a la idea de aldea global o globalización como ejercicio hegemónico de las tendencias capitalistas del presente. Según el profesor Quijano (2004), una nueva forma de dominación recorre el mundo: la de la globalización, y con ella la ideología del capital corporativo y del mercado mundial, que a su paso deja, entre otras derivaciones, un agresivo plan de intervenciones no solo político-militares sino también morales, jurídicas, productivas y biopolíticas; de otro lado está la huella ecológica que la especie humana está dejando actualmente sobre el planeta. Ahora bien, un asunto transversal a estas dos ideas tiene que ver 
con la posición política del individuo que intenta ser crítico con su entorno, entonces aparece en escena un elemento que no se ha considerado y es la forma como este individuo interviene en las decisiones de las colectividades de las que hace parte, en las que se confronta con obstáculos de gobernabilidad y participación ciudadana, que también han sido inteligentemente coartadas por la evolución misma de los sistemas políticos democráticos o, en el peor de los casos, por sistemas más coercitivos como las dictaduras y oligarquías, muy presentes en la historia política de América Latina.

Una de las reflexiones a las que apunta este escrito es determinar de qué manera el individuo asume el impacto que genera en su entorno y qué mecanismos tiene a su alcance para controlar los efectos de este impacto a mediano y a largo plazo. Para ello es necesario observar desde las dos perspectivas anteriormente mencionadas, con el fin de que las alternativas que se den a esta reflexión incidan en los componentes sociales y culturales que determinan el momento actual.

La otra reflexión gira alrededor de las iniciativas que las Naciones Unidas, y particularmente la Comisión Económica para América Latina y el Caribe - CEPAL, viene adelantando para la región, sobre los Objetivos de Desarrollo del Milenio - ODM -, que son el resultado de la Cumbre del Milenio realizada en septiembre de 2000, y que plantean los retos que para las naciones tiene el presente siglo, y se enfocan en ocho objetivos que buscan establecer las prioridades del desarrollo en el mundo en los ámbitos económico, social y ambiental. Este artículo se centra en el séptimo objetivo, que busca garantizar la sostenibilidad del medio ambiente: se analiza cómo los países latinoamericanos se están comprometiendo con el cumplimiento de este objetivo.

\section{Metodología}

Este artículo plantea un desarrollo metodológico de tipo documental con enfoque mixto, el cual ubica dos escenarios de análisis referidos en los resultados de investigación. Por tanto, en primer lugar se hace una evaluación del séptimo objetivo de los ODM a la luz de las políticas económicas de los países de la región; posteriormente se analizan algunas problemáticas que limitan el cumplimiento de los objetivos en la región, el impacto de las acciones de mejoramiento que implementan los estados a nivel global y el estancamiento subyacente sobre el control, la información y el ambiente que aún no permite evaluar y monitorear los avances logrados en los países de la región.

\section{Resultados}

\section{Diagnóstico del séptimo objetivo de desarrollo del milenio en América Latina}

En la CEPAL se ha desarrollado el enfoque de necesidades del hombre a partir de los Objetivos de Desarrollo del Milenio. Estos objetivos están planteados así:

1. Erradicar la pobreza extrema y el hambre;

2. Lograr la enseñanza primaria universal;

3. Promover la igualdad de género y el empoderamiento de la mujer; 


\section{Conterto osorio, w.}

4. Reducir la mortalidad de los niños menores de 5 años;

5. Mejorar la salud materna;

6. Combatir el VIH/SIDA, el paludismo y otras enfermedades;

7. Garantizar la sostenibilidad del medio ambiente, y

8. Fomentar una alianza mundial para el desarrollo.

Sobre el séptimo objetivo es preciso señalar lo siguiente:

[...] los impactos del cambio climático sobre los recursos hídricos podrían dificultar las tareas de universalizar el acceso a los servicios de agua potable y saneamiento. Los eventos extremos asociados al cambio climático afectarán negativamente a las personas que viven en asentamientos precarios (tugurios) y a los pueblos indígenas. (Naciones Unidas, 2010, p. 71)

El cambio climático alterará la calidad y productividad de los recursos naturales y ecosistemas; algunos de estos cambios podrían ser irreversibles, al reducir la diversidad biológica y acentuar la degradación ambiental. (Naciones Unidas, 2010, p. 71)

Las metas que se plantean para las naciones de acuerdo con la revisión hecha en 2007 son cuatro, a saber:

1. La integración de los principios del desarrollo sostenible en las políticas y programas nacionales, considerando en ellos la reversión de la pérdida de recursos del medio ambiente. 2. Reducir la pérdida de biodiversidad, alcanzando para el año 2010 una reducción significativa de la tasa de pérdida. 3. Reducir el porcentaje de personas sin acceso sostenible al agua potable y a los servicios básicos de saneamiento y, 4. Mejorar la vida de por lo menos 100 millones de habitantes de tugurios. (CEPAL, 2010)

De manera general se pueden conocer avances y retrocesos en la puesta en marcha de estos objetivos en los países de la región. En primer lugar, se ha dado un crecimiento importante en las áreas protegidas con fines de conservación, mayor cobertura de agua potable y servicios básicos de saneamiento y disminución para la población urbana que vive en tugurios, sin embargo, las emisiones de gases contaminantes han aumentado y aún se da una creciente pérdida de cobertura de bosques en el continente. (Naciones Unidas, 2010, p. 81)

Acerca del primer componente del séptimo objetivo, no se cuenta con datos oficiales de parte de los estados, lo único que se ha aportado para el análisis de este objetivo es el compromiso que tienen algunos estados en el avance de la legislación ambiental y el aumento en algunos países del presupuesto para la inversión ambiental y el gasto público relacionado con este ámbito (ver Tabla 2).

La pérdida de superficies boscosas sigue en aumento, principalmente a causa de la racionalidad económica de las economías neoliberales que persiguen la expansión de sus actividades productivas por encima de los intereses globales. Hasta el 2005 se había 
perdido un 7\% de la cobertura de bosques en la región, que equivale a 69 millones de hectáreas según datos de la FAO (2009).

Las emisiones de $\mathrm{CO}^{2}$ en la región han aumentado considerablemente en la década 20002010, principalmente por la quema de combustibles fósiles y en menor proporción, por el cambio en el uso de los suelos y la deforestación; sin embargo, gracias a los cambios en las políticas empresariales y a la nueva vanguardia de responsabilidad social empresarial, se ha dado un crecimiento en el sector de los servicios, en cuanto al desarrollo de nuevas tecnologías y nuevas tendencias sobre eco-eficiencia que han permitido reducir las emisiones de $\mathrm{CO}^{2}$.

De acuerdo con la CEPAL (2010), el principal reto en esta materia se relaciona con la gestión del uso de los suelos y con la deforestación, con lo cual la Amazonía representa un escenario de enorme importancia para la región, puesto que posee dos tercios de la biomasa forestal del mundo y ello podría contribuir notablemente a la mitigación del cambio climático por la captura de $\mathrm{CO}^{2}$.

Otro elemento es el consumo de sustancias que agotan la capa de ozono, no obstante gracias a los compromisos pactados a nivel internacional se ha logrado una disminución sostenida y se han dado avances en materia tecnológica y de mejoramiento de las relaciones entre los sectores público y privado en aras de mayor voluntad política para el cambio en esta materia. Por otro lado, a pesar de que América Latina no se encuentra entre las regiones más afectadas por la sobreexplotación de la pesca, algunas poblaciones se hallan en situación crítica por la modificación de los hábitat y la contaminación de los océanos. (Naciones Unidas, 2010, p. 198)

Finalmente, sobre este punto es necesario mencionar el manejo de los recursos hídricos, que representan para la región una de sus mayores riquezas, pero cuya distribución se encuentra bastante desigual, lo cual ha generado problemas políticos y sociales en la mayoría de países de la región, además, la falta de gestión en materia de conservación y mantenimiento de fuentes hídricas ha llevado a una creciente contaminación de los afluentes por causas antropogénicas y, en consecuencia, al agotamiento y al uso insostenible del recurso. (Naciones Unidas, 2010, p. 198)

Sobre la segunda meta, relacionada con la pérdida de biodiversidad, es necesario mencionar en primera instancia la gestión que se ha desarrollado, y que viene en aumento, en el manejo de áreas marinas y terrestres protegidas, gracias al interés de las naciones de valorar su patrimonio natural. Sin embargo, existen algunos problemas asociados a estas iniciativas y que atañen principalmente a las poblaciones de estas mismas áreas, sobre las cuales no se hace ningún tipo de inversión en el manejo comunitario de bosques, y no reciben beneficios por las certificaciones ambientales y por la vinculación de estas poblaciones con los planes de ordenamiento territorial. (Naciones Unidas, 2010, p. 202)

La tercera meta tiene que ver con la sostenibilidad del ambiente humano, sobre todo con relación al acceso al agua potable y a los servicios básicos de saneamiento. En este sentido 


\section{Conterto osorio, w.}

la región ha mostrado una interesante evolución en la cobertura de servicios básicos y de acceso a fuentes de agua potable, especialmente en las ciudades. Infortunadamente este esfuerzo no compensa la acelerada expanción que están teniendo las ciudades y este avance solo es posible de realizar en los sustratos de la sociedad que pueden cubrir sus necesidades básicas. Para otra gran proporción de habitantes de la urbe aún no existen estos privilegios; este problema obedece principalmente a asuntos relacionados con la propiedad, a las políticas macroeconómicas, al sistema financiero y a las condiciones estructurales de cada país. (Comité de Derechos Económicos, Sociales y Culturales de la ONU, 2002)

\section{Avances y retrocesos en la gestión ambiental en América Latina}

El elemento más importante que se debe revisar son los niveles de concentración de gases de efecto invernadero - GEI -. Estos gases se emiten a la atmósfera como producto de los procesos industriales en el mundo desarrollado; está demostrado que de persistir esta tendencia la temperatura de la atmósfera puede aumentar a niveles que afectarían la vida en el planeta (Naciones Unidas, 2010, p. 63). Bajo esta premisa, los países que se comprometieron en 1997 con el Protocolo de Kyoto en la Convención Marco de las Naciones Unidas sobre el Cambio Climático están generando acciones para mitigar este efecto, que puede traer potenciales implicaciones negativas en el desarrollo de los países. A pesar de que América Latina es una de las regiones que menos gases emite - ocupa el segundo lugar después de África -, sí es una de las que más puede sufrir los impactos del aumento de la temperatura, que se estima puede ser de $4^{\circ} \mathrm{C}$ en las zonas tropicales y las zonas templadas de la región, lo cual tendría graves consecuencias para su desarrollo ya que afectaría la producción de alimentos, las zonas semidesérticas y los recursos hidrográficos de los países. (Naciones Unidas, 2010, p. 71)

En la Tabla 1 se pueden observar algunas de las tendencias de los indicadores del séptimo objetivo de desarrollo del milenio en el continente y su variación de 1995 a 2005. 
Tabla 1. Indicadores del séptimo objetivo de desarrollo del milenio en América Latina

\begin{tabular}{|c|c|c|c|c|c|}
\hline $\begin{array}{l}\text { Indicador } \\
\text { séptimo } \\
\text { objetivo de } \\
\text { desarrollo del } \\
\text { milenio }\end{array}$ & $\begin{array}{l}\text { Nombre Indicador } \\
\text { séptimo objetivo de } \\
\text { desarrollo del milenio }\end{array}$ & $\begin{array}{l}\text { América } \\
\text { del Sur }\end{array}$ & El Caribe & Mesoamérica & $\begin{array}{l}\text { América } \\
\text { Latina y el } \\
\text { Caribe }\end{array}$ \\
\hline 7.1 & $\begin{array}{l}\text { Proporción de la } \\
\text { superficie cubierta por } \\
\text { bosques }\end{array}$ & $-6,71$ & 13,23 & $-10,35$ & $-6,97$ \\
\hline $7.2 \mathrm{a}$ & $\begin{array}{l}\text { Emisiones de dióxido } \\
\text { de carbono total }\end{array}$ & 55,70 & 41,14 & 19,06 & 40,82 \\
\hline $7.2 \mathrm{~b}$ & $\begin{array}{l}\text { Emisiones de dióxido } \\
\text { de carbono por capital }\end{array}$ & 22,33 & 18,18 & $-7,85$ & 10,66 \\
\hline $7.2 \mathrm{c}$ & $\begin{array}{l}\text { Emisiones de dióxido } \\
\text { de carbono por cada } \\
\text { dólar del PIB }\end{array}$ & $-5,47$ & $-8,87$ & $-27,94$ & $-14,29$ \\
\hline 7.3 & $\begin{array}{l}\text { Consumo de } \\
\text { sustancias que agotan } \\
\text { la capa de ozono }\end{array}$ & $-89,41$ & $-87,76$ & $-93,55$ & $-84,56$ \\
\hline 7.6 & $\begin{array}{l}\text { Proporción de las } \\
\text { áreas terrestres y } \\
\text { marinas protegidas }\end{array}$ & 121,73 & 39,34 & 127,19 & 119,95 \\
\hline 7.8 & $\begin{array}{l}\text { Proporción de la } \\
\text { población con acceso } \\
\text { a fuentes mejoradas } \\
\text { de abastecimiento de } \\
\text { agua potable }\end{array}$ & 9,93 & 8,86 & 16,01 & 9,94 \\
\hline 7.9 & $\begin{array}{l}\text { Proporción de } \\
\text { la población con } \\
\text { acceso a servicios } \\
\text { de saneamiento } \\
\text { mejorados }\end{array}$ & $-31,06$ & $-32,43$ & $-32,42$ & $-31,27$ \\
\hline 7.10 & $\begin{array}{l}\text { Proporción de la } \\
\text { población urbana que } \\
\text { vive en tugurios }\end{array}$ & $-31,06$ & $-32,43$ & $-32,42$ & $-31,27$ \\
\hline
\end{tabular}

Fuente. Comisión Económica para América Latina y el Caribe (CEPAL).

Los datos suministrados en la Tabla 1 muestran la proporción del aumento o la disminución en cifras negativas de cada uno de los indicadores del séptimo objetivo de desarrollo del milenio en el continente. A partir de esta mirada es importante conocer cuál es el compromiso institucional que asumen los países de la región a través de las instancias ambientales a cargo. La Tabla 2 da cuenta de cómo los países vienen aumentando de manera gradual su cuota de participación para mitigar el efecto de los GEI, con lo cual podrían mejorarse las acciones para disminuir los efectos contaminantes a largo plazo. 


\section{Conterto osorio, w.}

Tabla 2. Presupuesto total ejecutado en los países de la región (en dólares)

\begin{tabular}{|c|c|c|c|c|c|c|}
\hline País & 1995 & 2000 & 2005 & $\begin{array}{c}\text { Porcentajes } \\
\text { acumulados } \\
\text { de variación } \\
1995-2000\end{array}$ & $\begin{array}{l}\text { Porcentajes } \\
\text { acumulados } \\
\text { de variación } \\
2000-2005\end{array}$ & $\begin{array}{c}\text { Porcentajes } \\
\text { acumulados } \\
\text { de variación } \\
1995-2005\end{array}$ \\
\hline Argentina & 17342051 & 16878338 & 15349971 & $-2,6$ & $-9,0$ & $-11,4$ \\
\hline Uruguay & 1591269 & 3222016 & 1676786 & 102,4 & $-47,9$ & 5,3 \\
\hline Chile & 10375448 & 20224588 & 18341236 & 94,9 & $-9,3$ & 76,7 \\
\hline Guatemala & & & 4999332 & & & \\
\hline Brasil & & 315696239 & 490133036 & & 55,2 & \\
\hline México & 10725268015 & 1569965185 & 2612000848 & 1363,8 & 66,3 & 2335,3 \\
\hline Colombia & 47965823 & 16514081 & 79307549 & $-65,5$ & 380,2 & 65,3 \\
\hline Belice & & 126880 & 218041 & & 71,8 & \\
\hline
\end{tabular}

Fuente: Comisión Económica para América Latina y el Caribe (CEPAL), sobre la base de información de los minesterios o secretarías de medio ambiente de los respectivos países.

Sin embargo, este aumento en los niveles de participación no garantiza un compromiso fuerte de parte de las economías nacionales, por cuanto los recursos destinados para este objetivo no siempre se están incrementando gradualmente en el PIB. En la Figura 1 se presenta la participación que los países de la región tienen sobre su PIB, y de esta manera puede observarse con más precisión qué países asumen mayor compromiso económico con el objetivo.

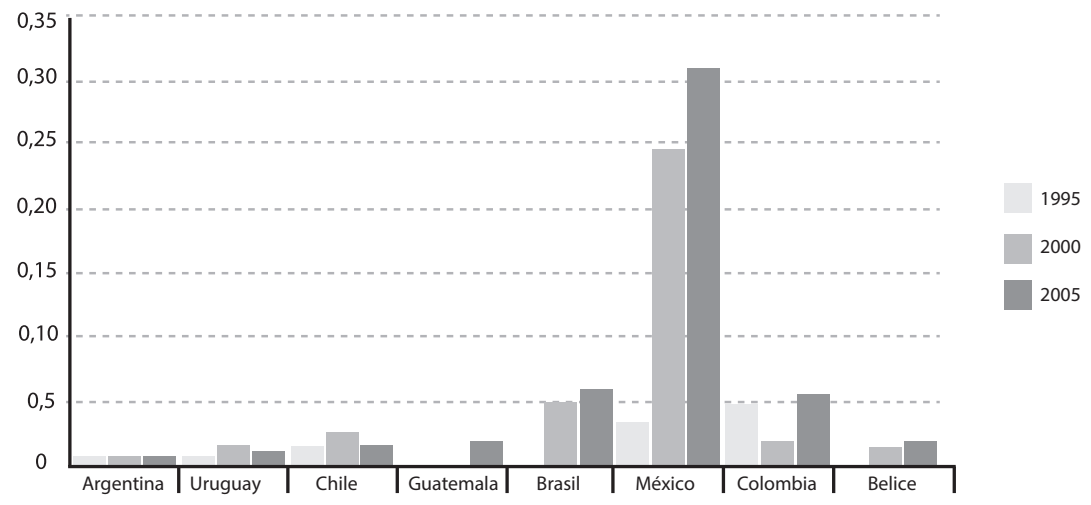

Figura 1. Presupuesto total ejecutado de ocho países de América Latina respecto al PIB corriente 1995, 2000 y 2005

Fuente: Comisión Económica para América Latina y el Caribe (Cepal), sobre la base de información de los minesterios o secretarías de medio ambiente de los respectivos países. 
Las principales inversiones que podrían hacer los países en este propósito tienen que ver con el aumento de las superficies boscosas para generar un equilibrio en las mediciones climáticas.

Algunos lineamientos que se requieren para mejorar los indicadores de superficies boscosas en América Latina tienen que ver con la adopción de medidas más eficaces para conocer el costo de la degradación y, en ese sentido, hacer una valoración de la rentabilidad de las empresas que hacen explotación de recursos del bosque, y equiparar esos resultados con aquellas actividades que fomentan el uso sostenible del recurso. (Naciones Unidas, 2010)

También se requiere una intervención continua al cuidado y sustentabilidad de los bosques nativos, que permita la utilización integral de los bienes forestales y madereros, y obviamente los servicios ambientales que provee la montaña. Del lado del Estado se debe fiscalizar y aplicar sanciones a las conductas ilegales, y para ello es importante el aumento de imágenes satelitales para monitorear la deforestación y proteger la biodiversidad. (Naciones Unidas, 2010, p. 195)

El bosque es el principal proveedor de materia prima para la industria farmacéutica, y otra importante inversión puede hacerse en la capacidad de desarrollar conocimientos y tecnología para aprovechar los recursos biológicos, especialmente en biotecnología. La biotecnología puede aportar un enfoque significativo en la producción de insumos agrícolas y en los procesos terapéuticos; la lucha contra las plagas, la fijación de nitrógeno atmosférico por los cereales (para disminuir la necesidad de fertilizantes), y el tratamiento biológico de tumores son distintos ejemplos de acciones "suaves', menos traumáticas para el ser humano y no erosionadoras de su entorno ambiental (Bercovich \& Katz, 1990, p. 22). Sin embargo, el régimen actual de propiedad sobre biotecnología no es muy favorable para la región a causa de la reglamentación con la que se suscriben los tratados de libre comercio, particularmente con los Estados Unidos. La Tabla 3 muestra el estado de las superficies boscosas en América Latina. 


\section{Conterto osorio, w.}

Tabla 3. América Latina y el Caribe: extensión y variaciones de la superficie boscosa

\begin{tabular}{|c|c|c|c|c|c|c|c|c|}
\hline \multirow{3}{*}{$\begin{array}{l}\text { Subregión } \\
\text { A. El caribe }\end{array}$} & \multicolumn{3}{|c|}{$\begin{array}{l}\text { Superficie de bosques } \\
\text { (en miles de ha) }\end{array}$} & \multirow{2}{*}{\multicolumn{2}{|c|}{$\begin{array}{c}\begin{array}{c}\text { Promedio anual } \\
\text { (en miles de ha) }\end{array} \\
\text { 19990-2000 2000-2005 }\end{array}$}} & \multirow{2}{*}{\multicolumn{2}{|c|}{$\begin{array}{c}\text { Variación promedio } \\
\text { anual } \\
\text { (en porcentajes) }\end{array}$}} & \multirow{3}{*}{$\begin{array}{c}\text { Acumulada } \\
\mathbf{1 9 9 9 0 -} \\
\mathbf{2 0 0 5} \\
\text { (en } \\
\text { porcentajes) } \\
13,23\end{array}$} \\
\hline & \multirow{2}{*}{$\begin{array}{l}1990 \\
4741\end{array}$} & \multirow{2}{*}{$\begin{array}{l}2000 \\
5098\end{array}$} & \multirow{2}{*}{$\begin{array}{l}2005 \\
5368\end{array}$} & & & & & \\
\hline & & & & 36 & 54 & 0,73 & 1,04 & \\
\hline $\begin{array}{l}\text { B. } \\
\text { Mesoamérica }\end{array}$ & 96655 & 89377 & 86649 & -728 & -546 & $-0,78$ & $-0,62$ & $-10,35$ \\
\hline $\begin{array}{l}\text { B.1. } \\
\text { Centroamérica }\end{array}$ & 27639 & 23837 & 22411 & -380 & -285 & $-1,47$ & $-1,23$ & $-18,92$ \\
\hline B.2. México & 69016 & 65540 & 64238 & -348 & -260 & $-0,52$ & $-0,40$ & -692 \\
\hline $\begin{array}{l}\text { C. América } \\
\text { del sur }\end{array}$ & 882727 & 844733 & 823477 & -3799 & -4252 & $-0,44$ & $-0,51$ & -671 \\
\hline $\begin{array}{l}\text { D. América } \\
\text { latina y el } \\
\text { caribe }\end{array}$ & 984123 & 939208 & 915494 & -4492 & -4743 & $-0,47$ & $-0,51$ & -697 \\
\hline El mundo & 4077291 & 1398861 & 3952025 & -8868 & -7317 & $-0,22$ & $-0,18$ & -307 \\
\hline $\begin{array}{l}\text { América latina } \\
\text { y el carice7 } \\
\text { mundo (en } \\
\text { porcentajes }\end{array}$ & 0,241 & 0,235 & 0,232 & & & & & \\
\hline
\end{tabular}

Fuente: Comisón Económica para América Latina y el Caribe (CEpal), base de estadisticas e indicadores ambientales (BADEIMA), sobre la base de cálculos realizados con la superficie nacional de bosques de evalucación de los recuersos forestales mundiales 2005 (FRA 2005) y la superficie terrestre nacional de bases de datos estadisticos de la FAO (FAOSTAT).

Es muy importante atender la demanda de energía a partir del crecimiento demográfico sin afectar las reservas de combustibles fósiles a mediano plazo y, por otro lado, incentivar el cambio hacia energías que puedan ser renovables y sostenibles, con el fin de poderse posicionar en el nuevo paradigma de comercio e inversión internacional bajo en carbono, ${ }^{1}$ con lo cual se podrá estimular un tipo de industria que utilice tipos de energía que emitan menos $\mathrm{CO}^{2}$, y de esta manera demostrar que aquellos procedimientos altos en generación de gases son más costosos que aquellos que implementan nuevas tecnologías más limpias.

1. También se le conoce como Economía de Bajo Carbono (LCE, por sus siglas en inglés), se trata de compensar a mediano y largo plazo las emisiones de gases de efecto invernadero, principalmente el dióxido de carbono por causas antrópicas, y aquellas naciones con emisiones intensivas de carbono o las que son densamente pobladas se están comprometiendo con las estrategias de mitigación del calentamiento global, neutralidad del carbono, geoingeniería y adaptaciones a los desafíos de una economía más sustentable. 
En la Figura 2 se pueden observar las inversiones que se hacen actualmente en la región para el desarrollo de este tipo de tecnologías, de modo consecuente con este propósito de la economía de bajo carbono.

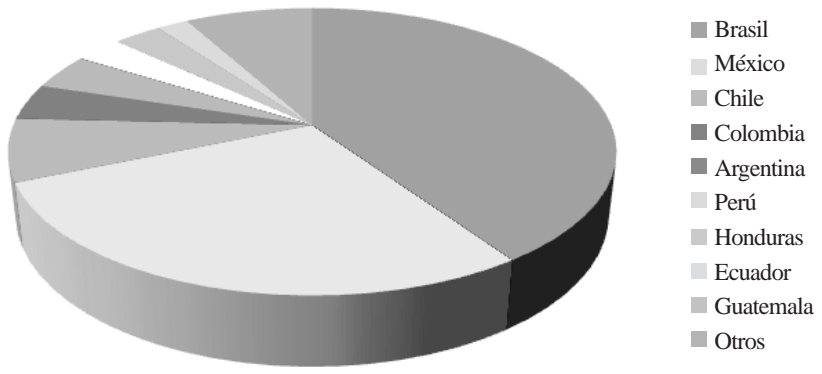

Figura 2. Cantidad de proyectos del mecanismo para un desarrollo limpio en América Latina y el Caribe (en porcentajes)

Fuente: Programa de las Naciones Unidas para el Medio Ambiente (PNUMA).

Se trata de una forma de financiación de la gestión ambiental que tiene que ver con los recursos financieros destinados a la sostenibilidad ambiental, como son los proyectos para un desarrollo limpio. Según se logra apreciar en la Figura 2, Brasil encabeza la lista de países por el número de proyectos para la reducción certificada de emisiones RCE, seguido de cerca por México. Lo anterior evidencia la importante gestión que se da en la región en esta materia. En la Figura 3 se muestra en detalle los proyectos sobre los que más se concentra la atención para las RCE en la región, y se presenta, como consecuencia del mayor grupo de proyectos presentados, un detalle de la oferta de energías renovables que la región desarrolla actualmente para favorecer la generación de energías más limpias y sostenibles para la humanidad.
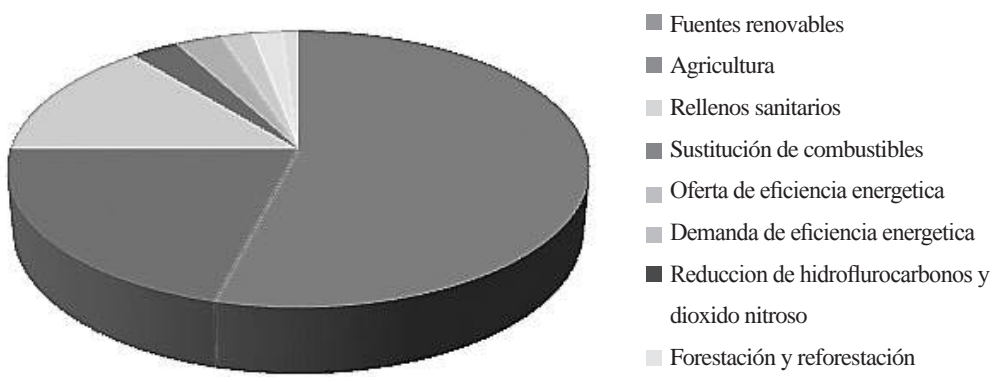

Figura 3. América Latina y el Caribe: Volumen de Reducciones Certificadas de Emisiones (RCE) por tipo de proyecto (en porcentajes)

Fuente: Programa de las Naciones Unidas para el Medio Ambiente (PNUMA). 


\section{Conterto osorio, w.}

En cuanto al acceso al agua potable y al saneamiento básico, las empresas prestadoras de servicios deben encontrar mecanismos de autofinanciación, dado que en los sectores más vulnerables de la sociedad existen fuertes limitaciones para cubrir los gastos asociados a la utilización de este tipo de servicios; no obstante, es posible avanzar en aspectos que tienen que ver con la regulación para mitigar asuntos que contribuyan a la disminución de los costos para el desarrollo del servicio, y por otro lado, establecer subsidios que garanticen a los sectores más pobres un consumo mínimo.

Sobre la proporción de población urbana que vive en tugurios, se hace necesario crear programas de financiamiento que permitan el acceso de la población a viviendas subsidiadas por el Estado. En un informe publicado por la - CEPAL -, denominado "Guía de la gestión urbana" (2003) se establecía que también para este propósito era importante crear mecanismos de participación de los distintos actores sociales para la toma de decisiones en aspectos como la salud, el empleo, la educación, el transporte, entre otros, que garanticen el mejoramiento gradual de las condiciones de vida de la población.

De alguna manera el movimiento ambiental a partir de los noventa ha permitido el establecimiento de la voluntad política de las principales naciones de la región y la iniciativa de la empresa privada de transformación en objetivos de responsabilidad social y ambiental; una coyuntura que también han sabido aprovechar las grandes corporaciones en el mundo, pues estas lograron hacer desaparecer los textos sobre sus propias responsabilidades ambientales y fortalecieron su dominio en el Tercer Mundo, convirtiendo el tema ecológico en publicidad para poder ejercer control en los recursos de los países en desarrollo, que según Ramonet (2009),

[...] las grandes firmas multinacionales se apropian de los recursos ambientales valiéndose de medios desmesurados, se aprovechan sin frenos ni escrúpulos de riquezas naturales que representan el bien común de la humanidad, con un pobre argumento de un mejor futuro para todos, pero sin considerar las consecuencias ambientales al largo plazo. Tomado de: Asociación por una tasa sobre las transacciones especulativas para ayuda a los ciudadanos - ATTAC -

Desde la caída del socialismo,

[...] se ha fomentado la idea falaz de que "el capitalismo salvaje" es la única estrategia válida de desarrollo y que junto con la apertura del mercado doméstico al mundo, procesos de privatización y no intervencionismo de estado, el "lesseferismo" en lo ambiental es lo que sigue. (Vásquez, 1999, p. 75)

De este modo, las naciones del norte abanderan una nueva estrategia de intervención económica, con la bandera del medio ambiente y el desarrollo sostenible, que en los círculos académicos se ha denominado neo-colonialismo. Este nos remite a pensar que la ecología es un hecho económico y político en el cual los países 
biodiversos son económicamente pobres y políticamente débiles; y al carecer de poder de decisión, el peso político y económico de los fuertes estará por encima de la importancia biológica y ecológica de los países tercermundistas.

Para intentar transformar esta corriente hegemónica, es preciso que cualquier proyecto de conservación del medio ambiente en la región pase por una reforma agraria, que a la vez brinde una triple distribución de la tierra, del ingreso y del poder. Con la reforma agraria se lograría: generación de empleo, aumento de la productividad de la mano de obra, ampliación del mercado interno, fomento de la producción de bienes de consumo y estabilidad de precios, mayor producción de bienes para exportar, reducción del éxodo a las ciudades, mejoramiento del nivel de vida, y verdadera democracia económica y política, ya que de acuerdo con los planteamientos del proferor Gilberto Vásquez (1999), el principal problema inflacionario radica en la tenencia o propiedad sobre los factores productivos; por otro lado, también considera, dentro de las reformas de parte del Estado, una redistribución del ingreso que mejore la capacidad de compra de las masas.

Otro aspecto importante en la transformación de las iniciativas ecológicas está directamente relacionado con la economía, y para ello es necesario citar las teorías de la Escuela Estructuralista, la cual

[...] sostiene que el escaso desarrollo de la región arranca de fallas capitalistas a nivel general y local, donde se dan fuertes concentraciones del ingreso $y$ de la riqueza incompatibles con el desarrollo y por otro lado problemas estructurales en el comercio internacional por la fuerte dependencia de los países industrializados que imponen las condiciones de comercio en la forma más benéfica para ellos. (Vásquez, 1999, p. 247)

El neoestructuralismo propone el aumento de la industrialización para sustituir las importaciones, con un proteccionismo selectivo de parte del Estado y de la empresa privada, además propone reformas como la agraria que se mencionó anteriormente, reformas tributarias, urbanas y de educación que conduzcan a la estabilidad política y que ayuden a estimular una democracia económica que pueda hacer peso a las oligarquías tan presentes en las naciones latinoamericanas, y que son las que sustentan el poder del latifundio, los monopolios y la concentración inequitativa del ingreso. (Vásquez, 1999, p. 250)

\section{Sobre la globalización}

La globalización, en palabras del profesor Quijano (2004), es un fenómeno multidimensional que se entrelaza en todos los aspectos de la cotidianidad moderna, que afecta las relaciones interpersonales y en consecuencia todas las formas de cultura que interactúan en la modernidad, por tanto, tal fenómeno no puede desconocerse para indagar sobre aspectos puntuales como el que convoca la temática de este artículo. 


\section{Conterto osorio, w.}

La pregunta por el individuo, la colectividad y las instituciones, inmersos en esta aldea global, es un factor que determina formas de comportamiento y de aceptación social, y cabe bien en estas circunstancias citar al profesor Quijano (2004) cuando plantea que, desde la teoría neoclásica, profundamente arraigada en la epistemología, la moral y la cosmología de occidente, resulta hoy contraproducente comparar este escenario, en un intento por entender otras economías, como ámbito de producción simbólica, productos culturales y productores culturales.

La economía mundial, en términos epistemológicos, plantea la reflexión sobre formas diferentes de valuación de la riqueza, formas más equitativas de distribución y un equilibrio medible y sustentable con el entorno inmediato, con lo cual principios y acciones determinantes en este propósito también pueden ser globales, moralmente aceptables y académicamente alcanzables, y es menester de los estados convocar a sus ciudadanos a apoyar iniciativas que salvaguarden los intereses colectivos sobre los individuales. Pero, en contraste, los estados contribuyen a difundir las tendencias de las mayorías y los objetivos de la homogenización de las acciones y los pensamientos de las sociedades, donde el primer afectado es el legado cultural que afirma y autodetermina la soberanía de los pueblos.

Actualmente se vive "una fase de capitalización o economización de la naturaleza, o en términos generales, se presencia un cambio y un salto cualitativo del capital, en el que la naturaleza adquiere una valoración positiva como fuente o reservorio de valor y riqueza" (Quijano, 2004, p. 15). En un sentido, este cambio suscita una idea de consideración del medio ambiente en términos de valoración económica, pero por otro lado da cuenta de cómo el lugar de asentamiento de cientos de sociedades que han habitado este planeta, y que desde los albores de la humanidad se han beneficiado de los recursos naturales y de los servicios ambientales que hoy esta humanidad disfruta, terminan siendo solo bienes que pueden ser comercializados. Sin embargo, es un punto de partida para la ecologización de la economía. ${ }^{2}$

Las decisiones que se tomen en torno a esta iniciativa afectan los aspectos socioeconómicos y políticos de las naciones, y en consecuencia facilitan la implementación de políticas públicas que favorecen el estudio y la profundización en el reconocimiento del patrimonio natural de los países. La biodiversidad, por ejemplo, se presenta como uno de los renglones más importantes por revisar, dado que en el futuro dinamizará, en sus componentes, dinámicas del capital; en la medida en que pueda ser medible y valorable en términos monetarios y determine, en últimas, una fuente de riqueza.

En consecuencia, es necesario evaluar experiencias exitosas de manejo de los recursos y de sostenibilidad a nivel local, y cómo lo local influencia las dinámicas y acciones en el plano nacional y de América Latina. Algunas de esas experiencias locales se pueden observar en las Reservas Naturales de la Sociedad Civil, en los

2. Esta frase se ha utilizado en distintos escenarios que buscan desarrollar formas alternativas de desarrollo económico que sean amigables con el medio ambiente. 
asentamientos colectivos y en las iniciativas "verdes" de algunos municipios del país, como formas de resistencia al modelo hegemónico de la economía y una nueva manera de interpretar las relaciones comerciales.

Estas iniciativas locales se pueden dar a conocer en el mundo a través del mismo fenómeno de la globalización:

La globalización constituye el estudio supremo de la internacionalización, la amplificación en "sistema mundo" de todos los lugares y de todos los individuos aunque en grados diversos. En este sentido, con la unificación del planeta, la tierra se vuelve un solo y "único" mundo, y se asiste a una re-fundación de la totalidad de la tierra. (Quijano, 2004, p. 20)

El objetivo sería apalancar diseños y modelos amigables con el medio ambiente que también pueden generar riqueza y que tienen la apuesta de ser sostenibles.

No obstante, la globalización establece controles sobre los procesos globales e interviene en la cultura, la economía y más directamente sobre la política de las naciones, y las soluciones alternativas siguen estando a la sombra de las directrices de la biopolítica de las grandes corporaciones en el mundo, y como cita el profesor Quijano, "la economía también está compuesta de sistemas de producción, juegos de verdad, relaciones de poder y de significación. Evidentemente, la economía no es solo ni siquiera principalmente una entidad material, es ante todo una producción cultural”. (Quijano, 2004, p. 22)

Los principales cambios en el diseño de los esquemas de transformación de la economía, y más aún de las sociedades, están principalmente arraigados en sus patrones culturales, con lo cual, para establecer cambios en los patrones de comportamiento de las colectividades hay que trabajar inicialmente en las representaciones culturales de los pueblos, en fortalecer los patrones de identidad, en sus relaciones sociales y cómo estas subjetividades interactúan con el mundo.

\section{Sobre el control, la información y el ambiente}

Es necesario abordar otros aspectos en la reflexión sobre medidas locales que puedan hacer frente a propósitos más amigables con el medio ambiente y que, como se viene desarrollando en párrafos anteriores, genere riqueza. Para ello es importante ser coherentes con los desarrollos disciplinares que se han dado en distintas áreas del conocimiento y que para el saber contable constituyen un insumo del cual ya existen afortunadamente varios referentes, en este sentido es preciso mencionar elementos relacionados con el control de los recursos, la información económica y el medio ambiente.

Un primer escenario para explorar es la manera como se difunde la información en la actualidad, gracias a la tecnología, y que internet ha posibilitado el acceso ilimitado de datos y de experiencias que se pueden aplicar a todo nivel, fenómenos locales 


\section{Contekto osorio, w.}

de resistencia sobre el actual modelo hegemónico se difunden con una celeridad y fuerza como antes no lo había experimentado la humanidad, y en este punto es interesante citar el movimiento de los Indignados en España. ${ }^{3}$ El bienestar colectivo y los intereses de una vida digna son móviles determinantes en la actualidad para convocar iniciativas populares a través de las redes sociales, y el país no es ajeno a esa realidad, también es posible a través de estos medios difundir desarrollos académicos que den cuenta de resultados y avances para mejorar los niveles de satisfacción en las necesidades básicas de los seres humanos; es un escenario que se puede trabajar en red, pero con los mismos criterios de objetividad y rigurosidad con que se hace en la academia, divulgando los resultados de las investigaciones y generando nuevas preguntas de investigación.

Uno de los elementos que se debe informar y difundir tiene que ver con la seguridad alimentaria para las actuales generaciones y este punto requiere toda la atención posible desde todas las esferas, pues "antes del presente siglo la producción natural de alimentos, la capacidad del ecosistema para purificar desechos y el incipiente desarrollo industrial que aportaba bajos niveles contaminantes, hacían pensar en la infinidad de valiosos recursos naturales" (Franco, 2009, p. 34). Pero en el presente siglo la realidad es bien distinta; la producción actual de alimentos no es suficiente para cubrir la demanda de los crecientes niveles demográficos, la capacidad de absorción de desechos de la naturaleza está excedida, y los niveles de contaminación han llegado a proporciones nunca antes experimentadas en la historia de la humanidad.

Las teorías antropocéntrica y ecocéntrica permiten a la contabilidad dirigir sus acciones a nuevas esferas del conocimiento, para identificar las directrices que permiten a cada teoría dar solución desde su óptica a los conflictos ambientales sobre la base de un desarrollo sustentable, ya que es ilógico que la humanidad deje de crecer económicamente, como también lo es el creer que los recursos naturales y el medio ambiente son infinitos. (Tobón \& Serna, 1999, p. 33)

El profesor Rafael Franco cita a Thomas Robert Malthus, quien en el siglo XVIII planteó los efectos del crecimiento de la población, y expuso además cómo la producción y el capital serían insuficientes para atender la demanda de las necesidades básicas de los seres humanos (Franco, 2009, p. 34); pero sus tesis no tuvieron resonancia en su época y hoy se viven en la cotidianidad muchas de las preocupaciones que el autor tenía hace más de doscientos años. En su época el clérigo "estableció una relación de disparidad entre población humana y recursos para sustentarla, y sostenía que mientras la población crece en progresión geométrica, los alimentos solo crecen en progresión aritmética" (Vásquez, 1999, p. 79), con lo cual el capitalismo clásico justifica la pobreza con las teorías malthusianas, pero esto es solo una pobre interpretación de la visión de este autor, las ideas malthusianas van más allá de la implementación de un modelo, contemplan hábitos sociales y comportamientos culturales.

3. El movimiento de los indignados es un movimiento ciudadano formado a raíz de los abusos de la clase dirigente española y como respuesta a una serie de protestas pacíficas, con la intención de promover una democracia más participativa, alejada del fuerzas políticas tradicionales y del dominio de bancos y corporaciones. 
Actualmente les corresponde a los países determinar su participación en la configuración de los cambios necesarios para constituir una realidad diferente que esté a la medida de las necesidades de la humanidad, y con ello tomar posición como ciudadanos críticos para establecer políticas públicas que favorezcan los intereses básicos de las naciones.

La protección de la diversidad biológica es uno de los primeros objetivos para empezar a dejar un mejor porvenir y con ello, como renglón determinante, garantizar la seguridad alimentaria como bastión de la soberanía de los pueblos. Para proteger esta riqueza es menester medirla y valorarla, de ahí que sea necesario generar los instrumentos y herramientas que permitan conocer los elementos cualitativos que la componen, una caracterización de los recursos naturales que dé cuenta de la composición físico-química de sus elementos y el aporte que hacen al establecimiento de la vida; es igualmente necesario tener un inventario con georreferenciación de los recursos con los que los países cuentan para tener insumos cuantitativos que se puedan monitorear.

Sobre estos objetivos se deben fundar las políticas públicas relacionadas con medio ambiente y desarrollo, que puedan optimizar al máximo los recursos no renovables y el sostenimiento, sin mayores riesgos ambientales, de los recursos renovables. Para ello es necesario fijar unos indicadores que den cuenta de la gestión del manejo de estos recursos a mediano y largo plazo.

La producción de bienes y servicios requiere insumos del medio ambiente natural y a su vez hace sentir en él sus efectos. Estos efectos son, en particular, el agotamiento de los recursos y la producción de desechos que se descargan en el medio ambiente. La contaminación ocurre cuando estos desechos perturban los sistemas naturales, en particular los que son importantes para el bienestar humano (por ejemplo el aire y el agua). Si se considera al medio ambiente natural como stock de capital natural y a sus usos en beneficio del género humano como servicios procedentes de ese stock, la utilización del medio ambiente natural para la actividad económica se puede contabilizar, en principio, de la misma manera que la utilización de otros tipos de capital (por ejemplo el capital manufacturado que incluye máquinas, edificios e infraestructura) y los productos asi obtenidos. (Naciones Unidas, 2013, p. 2)

Esta forma de medición del capital se denomina capital natural, un término que empezó a difundirse desde la economía ambiental y que está directamente relacionado con los conceptos de sostenibilidad débil y fuerte, ${ }^{4}$ y las formas de sustitución del mismo: "el capital natural se puede definir como el conjunto de dinámicas valiosas que la naturaleza provee a los seres humanos, que incluye la formación y regeneración de los recursos naturales y de donde fluyen constantemente una serie de servicios ambientales". (Isa, Ortúzar \& Quiroga, 2005, p. 12)

4. Es importante aclarar que la sostenibilidad débil es una apreciación sobre la manera de compensar el agotamiento del capital natural con otras formas de capital, mientras que la sostenibilidad fuerte sugiere la conservación del inventario del capital. 


\section{Conterto osorio, w.}

En esa lógica, el capital natural comprende la constitución de los recursos naturales (los bosques, los suelos, los terrenos para los asentamientos humanos, los afluentes de agua, los minerales, entre otros; pero además constituye los servicios ambientales que se refieren al manejo del clima, a la composición de la atmósfera para el aire que respiran las especies, el ciclo hidrológico del agua que se consume, la absorción de residuos, los distintos nutrientes necesarios para la vida, la polinización de los alimentos, el paisaje, entre otros. "Y como cualquier forma del capital es posible medirlo, cuantificarlo y sobre todo controlarlo". (Isa, Ortúzar \& Quiroga, 2005, p. 12)

Ello genera entonces la necesidad de diseñar un sistema de control que a través de procesos, matrices, informes y proyecciones dé cuenta del estado del capital natural en el tiempo. Parte de la solución está en definir mecanismos y metodologías de medición, valoración y control que permitan subsanar las deficiencias del sistema económico y contable. (Tobón \& Serna, 1999, p. 34)

Con este propósito se han puesto en marcha varios enfoques que pretenden desarrollar esquemas de contabilidad ambiental, para generar la información necesaria y hacer más efectiva la gestión ambiental. Existe el enfoque del World Resources Institute, el enfoque de la contabilidad ambiental como instrumento de gestión y el enfoque de las Naciones Unidas.

Por cuestiones de integralidad del modelo, en este documento se quiere explicar el enfoque de las Naciones Unidas, el cual sugiere la implementación de un sistema de información que integre los elementos contables y económicos que tienen relación con la gestión medioambiental. "La necesidad de una contabilidad integrada del medio ambiente y la economía se debe a la importancia crítica que tienen las funciones ambientales para el desempeño económico de un país y para el bienestar de su población" (Naciones Unidas, 2013, p. 29). Las funciones hacen relación al suministro de los recursos naturales, a las funciones de sumideros para la absorción de desechos y la función de servicios ambientales, de los que ya se hizo mención anteriormente.

El modelo de las Naciones Unidas se conoce como Sistema de Contabilidad Ambiental y Económica Integrada - SCAEI - (en inglés IEEA: Integrated Environmental and Economic Accounting). Se trata de una estructura para medir el desarrollo sostenible a partir de la medición de las tres funciones señaladas anteriormente y que se integra al Sistema de Cuentas Nacionales - SCN - de cada país, con lo cual podría incidir en las políticas macroeconómicas de los Estados; sinembargo el sistema no se encuentra totalmente implementado y muchas partes del manual aún se encuentran en desarrollo.

En América Latina solo dos países aplican el sistema, México y Colombia, y otros cuantos países empiezan a desarrollar en su sistema de cuentas nacionales la implementación de cuentas ambientales. En la Tabla 4 se puede observar la aplicación de cuentas ambientales en la región. 
Tabla 4. Aplicación de cuentas ambientales en América Latina

\begin{tabular}{|c|c|c|c|c|}
\hline $\begin{array}{l}\text { Países que están } \\
\text { elaborando C.A. }\end{array}$ & $\begin{array}{l}\text { Países con } \\
\text { proyectos para } \\
\text { elaborar C.A. }\end{array}$ & $\begin{array}{l}\text { Países que } \\
\text { tuvieron } \\
\text { proyectos y no } \\
\text { los concretaron }\end{array}$ & $\begin{array}{c}\text { Países que no } \\
\text { tienen proyectos } \\
\text { para elaborar C.A. }\end{array}$ & $\begin{array}{l}\text { Países que } \\
\text { dejaron de } \\
\text { elaborar C.A. }\end{array}$ \\
\hline Colombia & Argentina & Argentina & Cuba & Chile \\
\hline México & Honduras & Bolivia & Dominica & Costa Rica \\
\hline \multirow[t]{7}{*}{ Nicaragua } & Panamá & Brasil & Ecuador & \\
\hline & $\begin{array}{l}\text { República } \\
\text { Dominicana }\end{array}$ & & El Salvador & \\
\hline & & & Guatemala & \\
\hline & & & Jamaica & \\
\hline & & & Perú & \\
\hline & & & $\begin{array}{l}\text { ST. Kitts \& } \\
\text { Nevis }\end{array}$ & \\
\hline & & & Tinidad y Toago & \\
\hline
\end{tabular}

Fuente: Comisión Económica para América Latina y el Caribe - CEPAL -, Cuentas Ambientales en los países de América Latina y el Caribe: Estado de Situación.

No obstante, de acuerdo a lo formulado por el profesor Rafael Franco:

Es factible realizar algunos controles sin apelar a la existencia de cuentas ambientales. Tales procedimientos de control se pueden sintetizar en evaluación del control interno e indicadores de calidad ambiental, aunque en tiempos recientes se han desarrollado técnicas como las matrices verificatorias que permiten determinar el nivel de cumplimiento de disposiciones legales, técnicas o administrativas sobre los recursos naturales. (2009, p. 37)

Estas técnicas se refieren a la implementación de unos patrones de verificación que, para el caso, se refieren a los recursos naturales y los servicios ambientales, y, por otro lado, unas variables que puedan ser medibles.

La base de la medición, permite establecer las dimensiones del objeto del estudio, como son: las medidas de área, la capacidad de reproducción o de regeneración, la longitud, medidas compuestas como: características intrínsecas del recurso vs tiempo de recuperación, ritmo de destrucción, características del ecosistema, entre otros. (Tobón \& Serna, 1999, p. 34)

Estas variables deben ser consecuentes con la normatividad ambiental propia de cada país; algunas de estas variables deben considerar los niveles de contaminación y disponibilidad de los recursos. Para la aplicación de estas técnicas no es preciso el montaje de las cuentas ambientales, se requiere solamente la voluntad política 


\section{Contekto osorio, w.}

de los dirigentes gubernamentales y la conformación de equipos integrados por la sociedad civil y las instituciones para la fundamentación y montaje de las herramientas y las variables.

Apelando al argumento constitucional de que el fin último del Estado es la satisfacción de las necesidades básicas esenciales (Franco, 2009, p. 38), es posible establecer unos equipos interinstitucionales para el montaje de estas matrices. El profesor Rafael Franco hace una ambientación general de cómo pueden construirse estas matrices a la luz del control integral, ${ }^{5}$ y de esta manera dar el primer paso en la instrumentalización de los mecanismos de gestión ambiental al alcance de los contables del país.

El paso siguiente se relaciona con la valoración de los recursos y los servicios, con lo cual aquí ya es necesario volver al SCAEI, en cuyo desarrollo procedimental ha establecido tres tipos de cuentas para la contabilización de las partidas ambientales. Una de ellas se refiere a las cuentas de tipo estructural o las cuentas físicas, es decir que de acuerdo al agotamiento o la degradación del recurso serán cuentas de cantidad o de calidad. Las cuentas de tipo funcional dan lugar a las cuentas de gastos ambientales e inversiones realizadas en proyectos de mejoramiento de las condiciones ambientales y, finalmente, las cuentas de sostenibilidad, que requieren un cambio estructural del modelo de desarrollo impuesto por los países industrializados para darle una orientación desde una economía ecológica que aún está en construcción.

Uno de los principales problemas con los que se encuentran actualmente las cuentas estructurales o cuentas físicas tiene que ver con la valoración de los recursos naturales en términos monetarios:

[...] en este orden de ideas, el valor que una cosa puede adquirir, debe ser aquel que el mercado ha asumido para tal, es decir, el valor monetario. Aquellos bienes que son externos al mercado y que por lo tanto no poseen un valor determinado, como lo son el medio ambiente y los recursos naturales son tratados como "externalidades" y se desliga de ellos su "valor intrínseco" con los valores monetarios. (Tobón \& Serna, 1999, p. 35)

Para la contabilidad, un primer objetivo por alcanzar en el mediano plazo es el de la valoración ambiental, ya que las decisiones en esta materia se siguen tomando sobre la base de datos históricos y estadísticas ambientales que en la mayoría de los casos no consultan la realidad ambiental de la región y mucho menos la del país.

El control se hace una vez se obtiene la información generada en las etapas de medición y valoración y se hace un análisis y evaluación de los aspectos de mayor relevancia y sobre los cuales el hombre debe centrar su atención para prevenir, corregir y mitigar los daños ambientales. (Tobón \& Serna, 1999, p. 35)

5. En su artículo "Contabilidad y Control Ambiental", Franco hace énfasis en la conformación de los principales factores ambientales y los indicadores que apoyarían la gestión de control sobre los recursos naturales y los servicios ambientales, de la mano de la normatividad ambiental que existe en el país. 
El avance en el desarrollo de nuevas tecnologías en la medición de los recursos, los avances en términos de valoración y la consecuente evolución de los sistemas de información apoyada de las matrices de verificación de los recursos naturales y de los servicios ambientales, van a permitir la cualificación de los modelos de gestión en los países de América Latina.

\section{Conclusiones}

Si se consideran los esfuerzos en la región con respecto al aumento de la legislación ambiental y el engrosamiento de los presupuestos nacionales para el financiamiento del medio ambiente y el desarrollo sostenible, aún no es significativo el aporte de las naciones latinoamericanas a la mitigación de la huella ecológica que la humanidad está ocasionando sobre el planeta.

Aspectos como la pérdida de superficie boscosa en la región y la presencia de los Gases de Efecto Invernadero - GEI -, aún siguen en aumento, y no existe una implementación seria de políticas y acciones en función de estos dos aspectos, lo cual podría generar un desequilibrio importante en los indicadores del cambio climático.

Es importante resaltar el avance que se está dando en la región en el desarrollo de tecnologías y mecanismos para el acceso a fuentes de energía alternativas y que contribuye a la disminución del consumo de las reservas de combustibles fósiles, ya que en la región se cuenta aproximadamente con noventa proyectos para un desarrollo limpio. Esta iniciativa puede ayudar a disminuir uno de los factores que más altera el clima en razón a la emisión de $\mathrm{CO}^{2}$.

Desde la CEPAL se ha desarrollado una escuela estructuralista que puede hacer un contraste interesante a las políticas neoliberales que han regido la economía de los países de la región por más de 40 años, y que han demostrado su ineficacia en la distribución equitativa del ingreso y en el mejoramiento de la calidad de vida de los pueblos. LaEscuela Estructuralista propone un desarrollo desde dentro a través de cambios estructurales y transformaciones en la manera de ejercer el comercio.

La contabilidad como disciplina social puede contribuir de una manera determinante a la evolución de estas iniciativas. Ella permite que el desarrollo sostenible sea un propósito alcanzable y sobre todo medible a mediano y largo plazo, dado el carácter de control y monitoreo que puede ejercer sobre los recursos que mide, el avance en los datos sobre seguridad alimentaria, agotamiento y degradación de los recursos, y las proyecciones de los distintos indicadores de la gestión ambiental. 


\section{Conterto osorio, w.}

\section{Referencias bibliográficas}

Bercovich, N. \& Katz, J. (1990). Biotecnología y economía política; estudios del caso argentino. Comisión Económica para América Latina y el Caribe CEPAL-. Recuperado de: http://www.cepal.org/cgi-bin/getProd.asp?xml=/ publicaciones/xml/6/25596/P25596.xml\&xsl=/argentina/tpl/p9f.xsl\&base=/ argentina/tp1/top-bottom.xsl

CEPAL. (2003). Guía de la gestión urbana. Recuperado de http://www.cepal.org/ cgi-bin/getProd.asp?xml=/publicaciones/xml/8/14238/P14238.xml\&xsl=/ dmaah/tpl/p9f.xsl

CEPAL. (2010). Objetivos de Desarrollo del Milenio - Avances en la sostenibilidad ambiental del desarrollo en América Latina y el Caribe. Recuperado de http:// www.eclac.org/cgi-bin/getProd.asp? xml=/publicaciones/xml/6/38496/ P38496.xml\&xsl=/dmaah/tpl/p9f.xsl\&base=/dmaah/tpl/top-bottom.xsl

FAO. (2009). Estadísticas de la FAO. Recuperado de www.fao.org/statistics/es/

Franco, R. (2009). Contabilidad y control ambiental. Revista Lumina, 10, 33-58.

Isa, F., Ortúzar, M. \& Quiroga, R. (2005). Cuentas ambientales: conceptos, metodologías y avances en los países de America Latina y el Caribe. CEPAL - Comisión Económica para América Latina y El Caribe. Recuperado de http://www.cepal.org/publicaciones/xml/3/20993/lcl2229e.pdf

Leff, E. (1986). Los problemas del conocimiento y la perspectiva ambiental del desarrollo. México: Siglo XXI Editores.

Naciones Unidas. (2010). Objetivos de Desarrollo del Milenio: Avances en la sostenibilidad ambiental del desarrollo en América Latina y el Caribe. Recuperado de: http://www.eclac.cl/cgi-bin/getprod.asp?xml=/ publicaciones/xml/6/38496/P38496.xml\&xsl=/dmaah/

Naciones Unidas, Departamento de asuntos económicos y sociales. (2013). United Nations Statistics Division. Contabilidad Ambiental y Económica Integrada. Manual de operaciones. Recuperado de: http://unstats.un.org/ unsd/publication/SeriesF/SeriesF_78S.pdf

Quijano, O. (2004). Globalización, economía y cultura en la nueva oferta material y simbólica. Revita Lumina, 5, 9-32. 
Ramonet, I. (2009). Impacto de la globalización en los países en desarrollo. Recuperado de http://inabima.gob.do//descargas/bibliotecaFAIL/ Autores\%20Extranjeros/R/Ramonet,\%20Ignacio/Ramonet,\%20Ignacio\%20 -\%20Impacto\%20de\%20la\%20globalizacion\%20en\%20paises\%20en\%20 desarrollo\%20(2001).pdf

Tobón, L. \& Serna, H. (1999). Contabilidad y Medio Ambiente. Revista Lumina, 3, 31-4.

Vásquez, G. (1999). Aproximación a la Economía Política. Medellín: Universidad Pontificia Bolivariana.

\section{Para citar este artículo:}

Osorio, W.(2014). Aproximación política a la implementación de la gestión ambiental en América Latina desde los Objetivos de Desarrollo del Milenio. En-Contexto, 2, 27-49. 